\title{
Association of vigorous physical activity with age-related, site-specific loss of thigh muscle in women: the HIREGASAKI study
}

\author{
Madoka Ogawa, Naotoshi Mitsukawa, Mark Loftin, Takashi Abe
}

Objectives: To investigate the relationship between age-related, site-specific loss of thigh muscle and accelerometerdetermined daily physical activity.

Design: Cross-sectional study.

Methods: Fifty-one middle-aged and old women aged 52 to 83 years (mean age, 66.0 [SD 7.1] years) volunteered. Muscle thickness (MTH) was measured by B-mode ultrasound at 2 sites on the anterior (quadriceps [QF] 50\% of thigh length) and posterior (hamstring [HM] 50\% of thigh length) aspects of the thigh. MTH was expressed in terms relative to thigh length (MTH/L). Habitual daily activities on 30 consecutive days were recorded using an accelerometer. Exercise intensity was classified as sedentary to light, moderate, or vigorous. The total duration of each level of exercise intensity was calculated. Physical activity-related energy expenditure was estimated.

Results: Age was inversely correlated with the QF-50\% MTH/L ( $\mathrm{r}=-.286, P=.04)$, but not with the HM-50\% MTH/L $(\mathrm{r}=.102, P=.47)$. Age was also inversely correlated with the ratio of QF:HM 50\% MTH (r=-.330, $P=.018)$. Site-specific muscle loss of the thigh was observed in the present sample. There were no significant $(P>.05)$ correlations between the QF:HM 50\% MTH ratio and daily step counts ( $\mathrm{r}=.205)$, duration of light ( $\mathrm{r}=.204)$, moderate-intensity $(\mathrm{r}=.145)$ physical activity, and physical activity-related energy expenditure $(\mathrm{r}=.223)$. However, the QF:HM 50\% MTH ratio was significantly correlated with the duration of vigorous physical activity $(\mathrm{r}=.287, P=.04)$.

Conclusion: Age-related, site-specific loss of thigh muscle is associated with the amount of vigorous physical activity in middle-aged and old women.

(Journal of Trainology 2012;1:6-9)

Key words: sarcopenia $\boldsymbol{\square}$ aging $\mathbf{m}$ muscle mass $\boldsymbol{\square}$ ultrasound

S keletal muscle mass, which is mostly located in the lower body, constitutes approximately $40 \%$ of fat-free body mass in young adults ${ }^{1}$ but decreases with advancing age, especially after the fifth decade of life. ${ }^{2}$ These age-related changes lead to a decline of strength and power, a decrease in physical performance, and disability. ${ }^{3}$ Because the population of elderly people is growing in most countries, including Japan, the agerelated loss of skeletal muscle mass is likely to have a severe economic impact on the healthcare system.

Age-related loss of skeletal muscle mass (Sarcopenia) has been studied extensively by evaluating muscle volume, muscle cross-sectional area (CSA), and anatomical muscle thickness (MTH). ${ }^{4}$ Recently, Abe and colleagues demonstrated that age is associated with site-specific loss of skeletal muscle mass in Japanese men and women ${ }^{5,6}$ and in German men $^{7}$. Interestingly, in the anterior and posterior regions of the thigh, age-related muscle loss was observed in the quadriceps but not in the hamstrings and adductors. ${ }^{8}$ The etiology of sitespecific sarcopenia is multifactorial and complex. Several factors have been implicated such as declining anabolic hormone concentrations, nutritional deficiencies, chronic inflammation, and insulin resistance..$^{5,7}$ Decreased physical activity levels that occur with aging also contribute to sarcopenia. It is well established that hormonal receptors for mRNA and transcriptional activity are increased ${ }^{8}$ and that there is reduced insulin resistance ${ }^{9}$ in exercising muscles following an acute bout of exercise for sedentary individuals. There are therefore many important interrelationships between physical activity levels and other etiologic factors associated with health and aging that may contribute in varying degrees to the age-related loss of skeletal muscle mass in older populations. More recently, we examined the effect of habitual physical activity on age-related loss of skeletal muscle and found that there is site-specific loss in both active (more than once a week) and inactive old women. ${ }^{10}$ In that study, the subjects were classified into active and inactive groups based on their self-reported habitual physical activity. The effect of exercise intensity and duration of daily physical activities on site-specific loss of thigh muscle is unknown. However, muscle mass and strength losses associated with increasing age

Received November 7, 2011; accepted December 7, 2011

From the Graduate School of Frontier Sciences, University of Tokyo, Kashiwa, Japan (M.O), Active Aging Research Center, Toyo Gakuen University, Nagareyama, Japan (N.M., T.A.), Department of Health, Exercise Science, and Recreation Management, University of Mississippi, Oxford, MS, USA (M.L.), and Department of Health and Exercise Science, University of Oklahoma, Norman, OK, USA (T.A.).

Communicated by Kiyoshi Sanada, $\mathrm{PhD}$

Correspondence to Dr. Takashi Abe, 1401 Asp Ave, Room 12 HHC, Norman, Oklahoma. E-mail: t12abe@gmail.com

Journal of Trainology 2012;1:6-9 @2012 The Active Aging Research Center http://trainology.org/ 
can be attenuated by high-intensity resistance training. ${ }^{11}$ Therefore, we hypothesized that the site-specific loss of skeletal muscle mass is probably associated with the decline in exercise intensity of daily physical activities that occurs with increasing age. The purpose of the present study was to investigate the relationship between intensity and duration of daily physical activities and age-related thigh muscle loss in Japanese women.

\section{Methods}

Fifty-one middle-aged and old (aged 52-83 years) women were recruited through printed advertisement and by word of mouth. Before informed consent was obtained, a written description of the purpose and safety of the study was distributed to potential subjects, along with a lifestyle questionnaire. All subjects were free of overt chronic disease (eg, diabetes, angina, myocardial infarction, arthritic disorder, cancer, stroke, etc) as assessed by self-report. The study was conducted according to the Declaration of Helsinki and was approved by the Ethics Committee for Human Experiments of the Toyo Gakuien University, Japan.

Thigh muscle thickness was measured using B-mode ultrasound (Aloka SSD-2000, Tokyo, Japan) at 2 anatomic sites on the medial anterior (quadriceps [QF] 50\% of thigh length) and medial posterior (hamstrings [HM] 50\% of thigh length) aspects of the thigh. ${ }^{12}$ The measurements were taken while the subjects stood with their knees extended and relaxed. A 5-MHz scanning head was placed on the measurement site without depressing the dermal surface. The subcutaneous adipose tissue-muscle interface and muscle-bone interface were identified from the ultrasonic image, and the distance between the 2 interfaces was recorded as muscle thickness (MTH). Previous studies have reported that MTH is strongly correlated with anatomical muscle cross-sectional area. ${ }^{13} \mathrm{MTH}$ was expressed in terms relative to thigh length (MTH/L). Subcutaneous fat thickness was also measured using ultrasound at 6 sites, as described previously. ${ }^{12}$ Percent body fat was estimated from subcutaneous fat thickness using an ultrasound-derived prediction equation, ${ }^{12}$ and fat-free mass (FFM) was calculated. Body mass and standing height were measured to the nearest $0.1 \mathrm{~kg}$ and $0.1 \mathrm{~cm}$, respectively, by using a height scale and an electronic weight scale. Body mass index (BMI) was defined as body mass $(\mathrm{kg}) /$ height $^{2}\left(\mathrm{~m}^{2}\right)$.

Daytime physical activity was measured on 30 consecutive days after orientation, during which participants were familiarized with the use of an accelerometer (Lifecorder-EX, Suzuken Co. Ltd., Nagoya, Japan). Participants were shown how to attach the accelerometer on an elastic belt over their left or right hip and were instructed to record their daily physical activity for 30 consecutive days, beginning on the following day. If the participants did not use the accelerometer for a couple of days during the testing period, they were requested to record their activity for additional days. The accelerometer measures acceleration along the longitudinal axis every 4 second with an internal piezoelectric accelerometer and classifies the intensity into 10 activity levels from 0 to 9 based on the accelerometer signal. Level 0 (corresponding to $<0.06 \mathrm{G}$ ) denotes immobility, and levels 1 to 9 (corresponding to $\geq 0.06 \mathrm{G}$ ) denote subtle to vigorous movements. After the measurements were taken, the recorded activity level data were downloaded to a personal computer. In this study, exercise intensity was categorized into 1 of 3 activity levels based on the accelerometer signal: light (levels 1-3, <3 METs), moderate (Levels 4-6, 3-6 METs) and vigorous (levels 7-9, >6 METs). ${ }^{14}$ The total duration of each level of exercise intensity was calculated. Physical activityrelated energy expenditure and average daily step count were also determined using the accelerometer. ${ }^{14}$

Results are expressed as means and standard deviation for all variables. Linear regression analysis was used to assess the relationships between age and the quadriceps [QF] and hamstrings [HM] MTH as well as the QF:HM MTH ratio. In this study, we used the QF:HM MTH ratio for an index of age-related, site-specific loss of thigh muscle. Thus, simple and multiple linear regression analyses were performed on the QF:HM MTH ratio, habitual physical activity (total duration of 3 activity levels, physical activity-related energy expenditure, and average daily step count), and age.

\section{Results}

On average, age, standing height, body mass, percent fat, and FFM were 66 (7) years, $1.53(0.05) \mathrm{m}, 52.4(5.4) \mathrm{kg}, 26.6$ (5.1) \%, and $38.3(2.9) \mathrm{kg}$, respectively. The average BMI value of our subjects was $22.4(2.5) \mathrm{kg} / \mathrm{m}^{2}$, which was quite similar to that in the National Report in Japan $\left(22.5 \mathrm{~kg} / \mathrm{m}^{2}\right.$ for women aged $60-69$ years and $22.1 \mathrm{~kg} / \mathrm{m}^{2}$ for women aged $\geq 70$ years). The rate of regular sports activity, including walking (at least once a week), among the subjects was $78 \%$. The main types of sports activity were walking (45\%), tennis/table tennis (20\%), and yoga/tai chi (18\%).

The mean QF-50\% and HM-50\% MTH/L were 1.13 (0.14) and $1.50(0.17) \mathrm{mm} / \mathrm{cm}$, respectively, and the QF:HM MTH

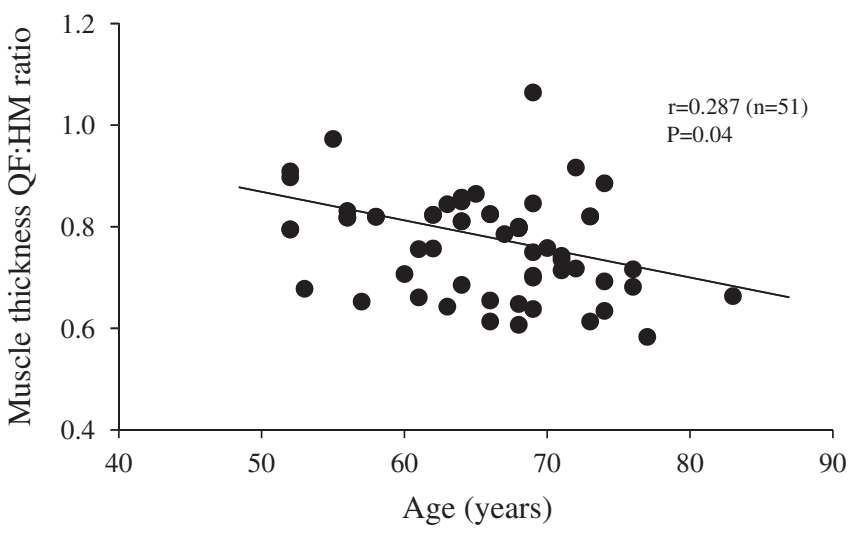

Figure 1. Relationship between age and ratio of $\mathrm{QF}: \mathrm{HM}$ $50 \%$ muscle thickness in women. QF, anterior thigh (quadriceps); HM, posterior thigh (hamstring/adductor). 
Table 1. Correlation coefficients between thigh muscle thickness and duration of light, moderate, and vigorous physical activities in women

\begin{tabular}{|c|c|c|c|c|c|}
\hline & \multicolumn{5}{|c|}{ Duration of Physical Activity (min/day) } \\
\hline & Light & Moderate & Vigorous & $\mathrm{M} \& \mathrm{~V}$ & Total \\
\hline \multicolumn{6}{|l|}{ Muscle thickness } \\
\hline Anterior (QF) $50 \%$ & 0.185 & 0.219 & $0.259 *$ & 0.228 & 0.244 \\
\hline Posterior (HM) 50\% & 0.066 & 0.031 & 0.089 & 0.002 & 0.032 \\
\hline QF:HM ratio & 0.204 & 0.145 & $0.287^{\dagger}$ & 0.162 & 0.228 \\
\hline
\end{tabular}

$\mathrm{M} \& \mathrm{~V}$, moderate and vigorous; Total, light, moderate and vigorous

$* P=.06 .{ }^{\dagger} P=.04$.

ratio was 0.76 (0.10). Daily step counts averaged 7827 (3184) steps and ranged from 2009 to 14,030 steps. The duration of light, moderate, and vigorous exercise during daytime physical activities were, respectively, 59 (20), 22 (17), and 2 (2) min per day. Age was inversely correlated with the QF-50\% $\mathrm{MTH} / \mathrm{L}(\mathrm{r}=-0.286, P=.04)$, but not the HM-50\% MTH/L $(\mathrm{r}=.102, P=.47)$. Age was also inversely correlated with the ratio of QF:HM 50\% MTH (r=-.330, $P=.018$ ) (Figure 1). There were no significant correlations between the QF:HM $50 \%$ MTH ratio and daily step counts $(\mathrm{r}=.205, P=.15)$, duration of light (r=.204, $P=.15)$, moderate ( $\mathrm{r}=.145, P=.31$ ), total $(\mathrm{r}=.228, P=.11)$ exercise, and physical activity-related energy expenditure $(\mathrm{r}=.223, P=.12)$. However, the QF:HM $50 \%$ MTH ratio was significantly correlated with the duration of vigorous physical activity $(\mathrm{r}=.287, P=.04)$ (Table 1$)$. The relationship between the QF:HM 50\% MTH ratio and vigorous physical activity was unchanged $(P=.037)$ after taking into account the possible effect of age.

\section{Discussion}

In the present study, the size of the quadriceps (QF) muscle, but not of the posterior (HM) muscle, was inversely correlated with age, and the QF:HM ratio was also inversely correlated with age. These results coincided with those of previous studies $^{7-10}$ and suggest that site-specific muscle loss of the thigh was observed in the present sample. Furthermore, the QF:HM ratio was positively associated with the duration of vigorous exercise during daytime physical activities. Our results suggest that a decrease in vigorous physical activity $(>6$ METs) is associated with age-related, site-specific loss of thigh muscle.

Starling et $\mathrm{al}^{15}$ reported no significant correlation between dual X-ray absorptiometry (DXA)-measured appendicular skeletal muscle mass and 9 days of uniaxial accelerometerdetermined physical activity, since age-related loss of appendicular skeletal muscle mass was observed. When estimating the yearly based physical activity of the elderly, $>30$ consecutive observation days are required to achieve $>90 \%$ reliability. ${ }^{16}$ Park et $\mathrm{al}^{17}$ reported that there were no significant correlations between DXA-measured leg muscle mass and year-averaged daily step count or duration of moderate intensity physical activity ( $>3$ METs) in women, although there is a significant correlation between leg muscle mass and duration of moderate physical activity ( $>3$ METs) in men. In the present study, we measured 30 consecutive days of physical activity in middle-aged and old women, with daily step count and duration of moderate physical activity having no significant correlation with thigh muscle size (MTH/L) or the QF:HM ratio. Duration of vigorous physical activity tended to be correlated with the QF-50\% MTH/L $(P=.06$, Table 1) and was significantly correlated with the QF:HM $\mathrm{MTH}$ ratio. Interestingly, a recent study observed positive associations between vigorous physical activity and lower body muscular strength tests in adolescents. ${ }^{18}$ The adolescents who were involved in resistance training had significantly higher muscular strength scores than youth in low and medium tertiles of physical activity, but no significant differences were found when they were compared with nonlifters from the highest tertile of physical activity. ${ }^{19}$ The results of the present study and the previous studies together suggest that duration of vigorous physical activity is an important predictor of, and may be strongly associated with, age-related, site-specific loss of thigh muscle.

In the present study, only 2 subjects reported performing regular resistance training. One of them had a relatively high duration of vigorous physical activity (4.4 min), but the other had very short duration of vigorous activity $(0.2 \mathrm{~min})$. The main types of sports/physical activity in this study were walking (45\%) and tennis/table tennis (20\%). Since muscle mass and strength losses associated with advancing age can be attenuated by high-intensity resistance training, ${ }^{11}$ other types of sports/physical activity, such as brisk walking or tennis/table tennis, may have provided sufficient muscular exercise to prevent the loss of quadriceps muscle mass. In addition, the duration of vigorous physical activity ( $>6$ METs) in the Japanese women ( $2 \mathrm{~min} /$ day $)$ in our study was similar to that reported in Canadian women $(3 \mathrm{~min} /$ day for women aged 40-59 years and $1 \mathrm{~min} /$ day for women aged 60-79 years), ${ }^{20}$ but both middle-aged and old women had a shorter duration compared with female adolescents (13.8 min/day). ${ }^{18}$ Therefore, even a small amount of regular vigorous physical activity may be associated with less age-related quadriceps muscle loss, although it is not clear how much vigorous physical activity is 
sufficient to have this effect.

\section{Conclusion}

Our results indicate that age-related, site-specific loss of thigh muscle is associated with the amount of vigorous physical activity in middle-aged and old Japanese women.

\section{Conflict of interest statement}

None of the authors had financial or personal conflict of interest with regard to this study.

\section{Acknowledgments}

The authors thank the individuals who participated in this study. This study was supported, in part, by Grant-in-aid (to NM) from the Japan Ministry of Education, Culture, Sports, Science, and Technology.

\section{References}

1. Abe T, Kearns CF, Fukunaga T. Sex differences in whole body skeletal muscle mass measured by magnetic resonance imaging and its distribution in young Japanese adults. Br J Sports Med 2003; 37: 436-440.

2. Porter MM, Vandervoort AA, Lexell J. Aging of human muscle: structure, function and adaptability. Scand J Med Sci Sports 1995; 5: 129-142.

3. Janssen I. Evolution of sarcopenia research. Appl Physiol Nutr Metab 2010; 35: 707-712.

4. Doherty TJ. Invited review: ageing and sarcopenia. J Appl Physiol 2003; 95: 1717-1727.

5. Abe T, Sakamaki M, Yasuda T et al. Age-related, site-specific muscle loss in 1507 Japanese men and women aged 20 to 95 years. J Sports Sci Med 2011; 10: 145-150.

6. Ogawa M, Yasuda T, Abe T. Component characteristics of thigh muscle volume in young and older healthy men. Clin Physiol Funct Imaging 2011. doi: 10.1111/j.1475-097X.2011.01057.x

7. Abe T, Kawakami Y, Kondo M et al. Comparison of ultrasound-measured age-related, site-specific muscle loss between healthy Japanese and German men. Clin Physiol Funct Imaging 2011; 31: 320-325.

8. Willoughby DS, Taylor L. Effects of sequential bouts of resistance exercise on androgen receptor expression. Med Sci Sports Exerc. 2004; 36: 1499-1506.

9. Black LE, Swan PD, Alvar BA. Effects of intensity and volume on insulin sensitivity during acute bouts of resistance training. J Strength Cond Res 2010; 24: 1109-1116.

10. Abe T, Kawakami Y, Bemben MG et al. Comparison of age-related, sitespecific muscle loss between young and old active and inactive Japanese women. J Geriatr Phys Ther 2011; 34: 168-173.

11. Steib S, Schoene D, Pfeifer K. Dose-response relationship of resistance training in older adults: a meta-analysis. Med Sci Sports Exerc 2010; 42: 902-914.

12. Abe T, Kondo M, Kawakami Y et al. Prediction equations for body composition of Japanese adults by B-mode ultrasound. Am J Hum Biol 1994; 6: 161-170.

13. Abe T, Kawakami Y, Suzuki Y et al. Effects of 20 days bed rest on muscle morphology. J Gravit Physiol 1997; 4: S10-S14.

14. Kumahara H, Schutz Y, Ayabe M et al. The use of uniaxial accelerometry for the assessment of physical-activity-related energy expenditure: a validation study against whole-body indirect calorimetry. Br J Nutr 2004; 91: 235-243.

15. Starling RD, Ades PA, Poehlman ET. Physical activity, protein intake, and appendicular skeletal muscle mass in older men. Am J Clin Nutr 1999; 70: 91-96.

16. Togo F, Watanabe E, Park $\mathrm{H}$ et al. How many days of pedometer use predict the annual activity of the elderly reliably? Med Sci Sports Exerc 2008; 40: 1058-1064.

17. Park H, Park S, Shephard RJ et al. Yearlong physical activity and sarcopenia in older adults: the Nakanojo Study. Eur J Appl Physiol 2010; 109: 953-961.

18. Moliner-Urdiales D, Ortega FB, Vicente-Rodriguez G et al. Association of physical activity with muscular strength and fat-free mass in adolescents: the HELENA study. Eur J Appl Physiol 2010; 109: 1119-1127.

19. Martinez-Gomez D, Welk GJ, Puertollano MA et al. Associations of physical activity with muscular fitness in adolescents. Scand J Med Sci Sports 2011; 21: 310-317.

20. Colley RC, Garriguet D, Janssen I et al. Physical activity of Canadian adults: accelerometer results from the 2007 to 2009 Canadian Health Measures Survey. Health Rep 2011; 22: 7-14. 